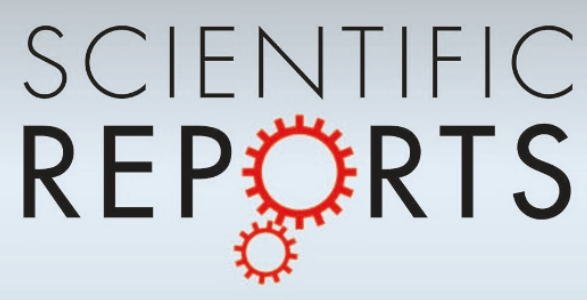

OPEN

SUBJECT AREAS:

THERMODYNAMICS

MECHANICAL ENGINEERING

COMPOSITES

APPLIED PHYSICS

Received

11 June 2013

Accepted

10 December 2013

Published

8 January 2014

Correspondence and requests for materials should be addressed to

X.B.L. (luoxb@hust. edu.cn)

\section{Local heating realization by reverse thermal cloak}

\author{
Run $\mathrm{Hu}^{\prime}$, Xuli Wei', Jinyan Hu' \& Xiaobing Luo'
}

\begin{abstract}
'State Key Laboratory of Coal Combustion, School of Energy and Power Engineering, Huazhong University of Science and Technology, Wuhan, 430074, China, ${ }^{2}$ School of Optical and Electronic Information, Huazhong University of Science and Technology, Wuhan, 430074, China.
\end{abstract}

Transformation thermodynamics, as one of the important branches among the extensions of transformation optics, has attracted plentiful attentions and interests recently. The result of transformation thermodynamics, or called as "thermal cloak", can realize isothermal region and hide objects from heat. In this paper, we presented the concept of "reverse thermal cloak" to correspond to the thermal cloak and made a simple engineering definition to identify them. By full-wave simulations, we verified that the reverse thermal cloak can concentrate heat and realize local heating. The performance of local heating depends on the anisotropic dispersion of the cloaking layer's thermal conductivity. Three-dimensional finite element simulations demonstrated that the reverse thermal cloak can be used to heat up objects. Besides pre-engineered metamaterials, such reverse thermal cloak can even be realized with homogenous materials by alternating spoke-like structure or Hashin coated-sphere structure.

N ith the born of transformation optics in 2006 $6^{1,2}$, it grows prosperously with a lot of attention and has turned into a subject of great interest since it not only provides a general method in coordinate transformation but also provides an effective design tool for manipulating electromagnetic wave ${ }^{3}$. In the past few years, transformation optics has been well developed beyond the domain of electromagnetic wave to plentiful extensions in other fields, including acoustic wave $e^{4,5}$, liquid wave $e^{6}$, matter wave $e^{7}$, and elastic wave ${ }^{8,9}$, etc. The results of these transformations, or called as cloaks, can be realized by pre-engineered metamaterials or even homogeneous natural materials with special-designed structures. One common type of cloaks is the core-shell structure, which maps a concealment region into a surrounding shell region with inhomogeneous distribution of constitutive parameters ${ }^{10-12}$. The core-shell structure, like the typical Hashin coated sphere structure, was initiatively described by Hashin ${ }^{9,13}$ as a model of composite comprised of spherical grains of one phase embedded in a matrix of another phase. Hashin pointed out how to design the phase materials and the corresponding fractions so that inserting the coated sphere into the matrix would not disturb the uniform electromagnetic field outside ${ }^{14}$.

Analogy to transformation optics, Guenneau et al. ${ }^{15}$ first extended the transformation methodology to the area of thermodynamics and proposed the concept of thermal cloak to channel the heat flow to realize a local isothermal region where the temperature gradient vanishes. Further studies ${ }^{16-19}$ demonstrated that thermal cloak can be realized with homogenous and finite thermal conductivity by simulations and experiments. More specifically, Han et al ${ }^{16}$ presented that thermal cloak can be realized by isotropic natural materials; Schittny et al. ${ }^{17}$ designed and manufactured a thermal cloak comprised of copper rings interlaid with plastic. From the view of guiding heat flux, Narayana and Sato ${ }^{18}$ did excellent and systematic work about controlling the heat flow by the engineering materials. By shielding, concentrating, and inverting the heat current, they experimentally showed that artificially engineered thermal materials can yield characteristics that go far beyond the conventional media.

The concept of thermal cloak, in fact, is derived from the optical invisible cloak, and it is aimed at hiding object from heat or rendering heat invisible in certain region. The thermal cloak can be achieved by anisotropic thermal conductivity distribution where the azimuthal component $\kappa_{\theta}^{\prime}$ is much larger than the radial component $\kappa_{r}^{\prime}$ $\left(\kappa_{\theta}^{\prime}>\kappa_{r}^{\prime}\right)$. Inspired by the previous work, one possible idea turns up that could we apply the thermal cloak reversely $\left(\kappa_{r}^{\prime}>\kappa_{\theta}^{\prime}\right)$ and realize a local heating region where the temperature gradient is large enough to heat up objects?

By utilizing the transformation thermodynamics and tailoring the dispersion of thermal conductivity, here we demonstrated that the reverse application of thermal cloak can efficiently realize a local heating region where the temperature gradient is more considerable than that of the outside region. Steady full-wave simulations show that the temperature profile in the central region strongly depends on the anisotropy of thermal conductivity. Transient simulations illustrate that heat diffuses faster in the left part of the reverse thermal cloak and slower 

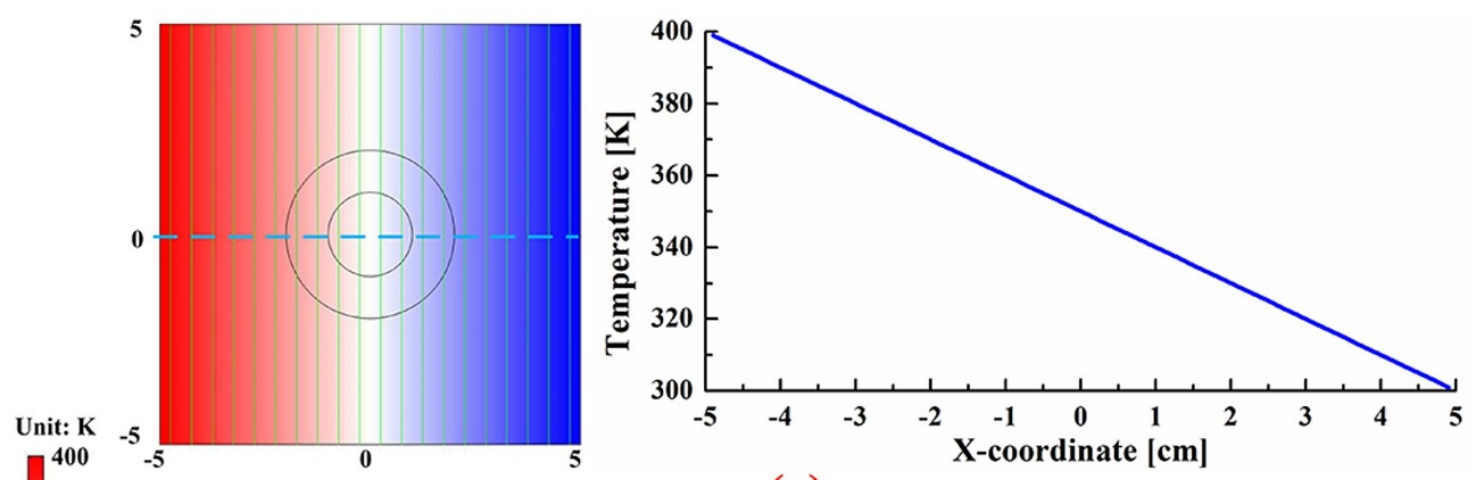

(a)
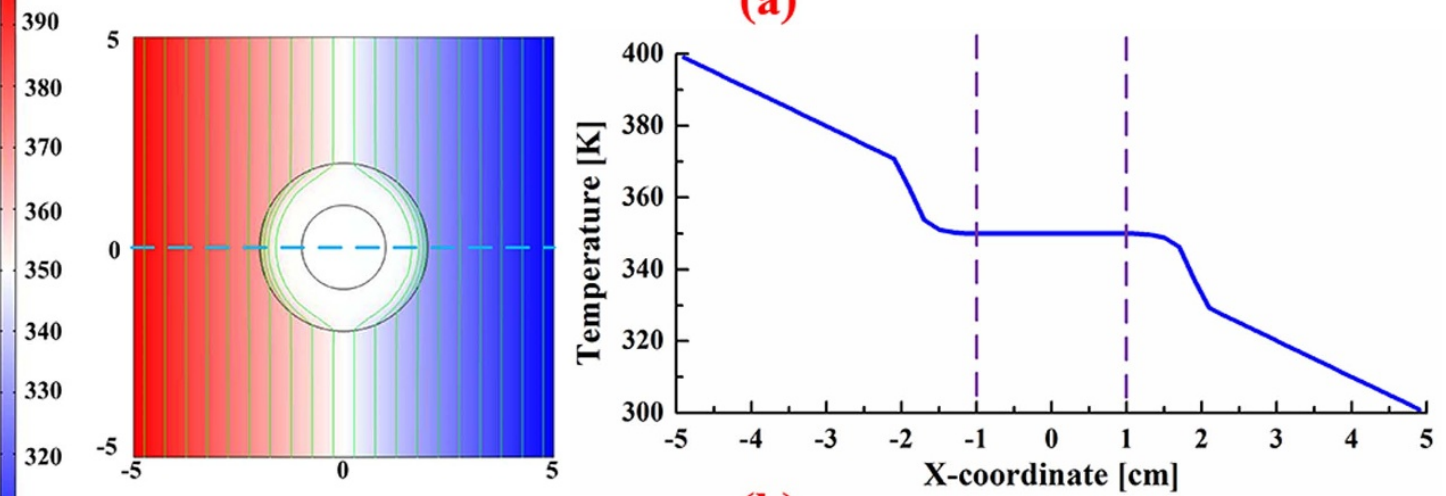

310

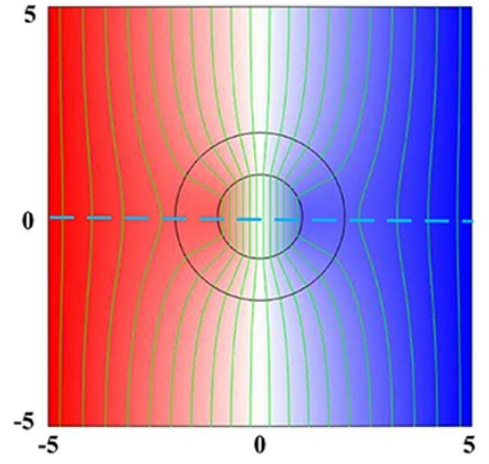

(b)

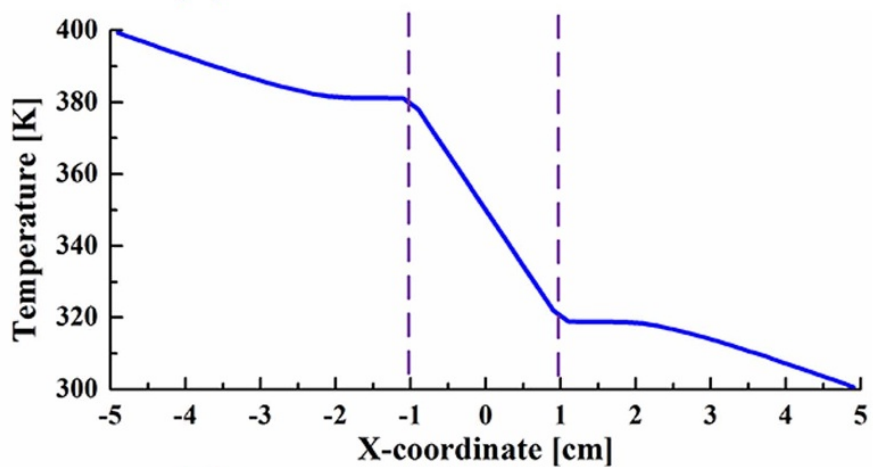

(c)

Figure 1 Temperature profile comparison among a homogenous plane, an annular thermal cloak and an annular reverse thermal cloak with constant boundary temperatures at $400 \mathrm{~K}$ and $300 \mathrm{~K}$ respectively: (a) a plane and the corresponding temperature curve of section line at $\mathrm{y}=0$; (b) a thermal cloak and the corresponding temperature curve of section line at $\mathrm{y}=0,\left(C=\kappa_{\mathrm{r}}^{\prime}=0.1, \kappa_{\theta}^{\prime}=10\right) ;(\mathrm{c})$ a reverse thermal cloak and the corresponding temperature curve of section line at $\mathrm{y}=\mathbf{0}\left(C=\boldsymbol{\kappa}_{\mathrm{r}}^{\prime}=\mathbf{1 0}, \boldsymbol{\kappa}_{\theta}^{\prime}=\mathbf{0 . 1}\right)$. Isotherms are illustrated in green color in panel. Cyan dash lines are the section lines.

in the right part, thus large temperature gradient is formed in the central region. By modeling a substrate consisting of reverse thermal cloak and assembling a cubic probe onto the central region, the corresponding temperature fields on the cubic probe show that local heating effect was enhanced with reverse thermal cloak. Two possible methods were also presented to realize such reverse thermal cloak.

\section{Results}

With transformation thermodynamics, we derived that the radial thermal conductivity $\kappa_{r}^{\prime}$ and the azimuthal thermal conductivity $\kappa_{\theta}^{\prime}$ are the inverse of each other, as shown in Eq. (1).

$$
\kappa_{r}^{\prime} \kappa_{\theta}^{\prime}=1
$$

By defining $\kappa_{r}^{\prime}=1 / \kappa_{\theta}^{\prime}=C$, where $C$ is a constant, a cloak can be realized with constant thermal conductivity. For thermal cloaks, the constant $C$ is usually less than $1^{16}$, implying the radial conductivity $\kappa_{r}^{\prime}$ is much smaller than the azimuthal conductivity $\kappa_{\theta}^{\prime}$. The performance characteristics of thermal cloak are totally attributed to its anisotropic distribution of thermal conductivity. In this paper, we proposed the concept of "reverse thermal cloak" where $\kappa_{r}^{\prime}$ is much larger than $\kappa_{\theta}^{\prime}$, i.e. the constant $C$ is larger than 1 .

With full-wave simulations based on finite element method (FEM), the steady-state performances of the cloaks were examined. The large commercial software package COMSOL Multiphysics was used to conduct the simulations. For comparison, a homogeneous plane with constant thermal conductivity was also simulated. In the simulations, the left and right boundaries were kept at constant temperature, i.e. $400 \mathrm{~K}$ and $300 \mathrm{~K}$, respectively. The top and bottom boundaries are thermally insulated. The interior and exterior radii of the annular thermal cloak (reverse thermal cloak) are $1 \mathrm{~cm}$ and $2 \mathrm{~cm}$, respectively. The dimensions of the whole domain are $10 \times 10 \mathrm{~cm}^{2}$. The thermal conductivities of the core region and the substrate are kept as $0.1 \mathrm{~W} /(\mathrm{m} \cdot \mathrm{K})$ and $1.0 \mathrm{~W} /(\mathrm{m} \cdot \mathrm{K})$, respectively. 


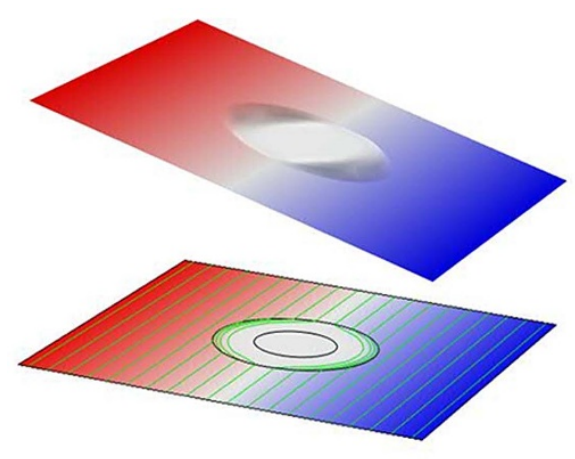

(a)

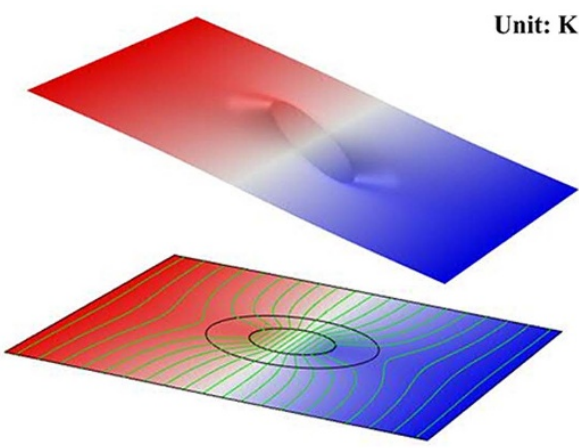

(b)

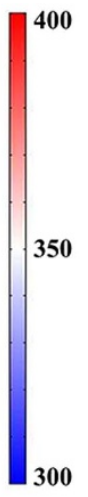

00

Figure $2 \mid$ Visual illustration of the temperature profiles of (a) a thermal cloak and (b) a reverse thermal cloak.

Fig. 1 shows the temperature profiles and corresponding temperature curves of the section lines on a homogenous plane, a thermal cloak and a reverse thermal cloak, respectively. From Fig. 1(a), the temperature profile of the homogenous plane is uniform and the temperature gradient is constant throughout the plane. From Fig. 1(b), the temperature profiles outside the thermal cloak are all the same with Fig. 1(a), but the heat tends to be away from the inner core region and travels close to the outside edge of the thermal cloak $\left(C=\kappa_{r}^{\prime}=0.1 \mathrm{~W} /(\mathrm{m} \cdot \mathrm{K}), \kappa_{\theta}^{\prime}=10 \mathrm{~W} /(\mathrm{m} \cdot \mathrm{K})\right)$. From both the temperature contour and the temperature gradient of the section line, we can see that thermal cloak can realize an isothermal region in the central domain. This implies that any object placed in the interior region appears to be concealed since the outside heat flow looks like as if there is no disturbance at all in the central region (the temperature gradient outside the thermal cloak is the same with that in Fig. 1(a)). This is the reason and origin of the definition of "thermal cloak". Fig. 1(c) shows that temperature field of the reverse thermal cloak in which we adjust the constant $C$ to $10\left(C=\kappa_{r}^{\prime}=10 \mathrm{~W} /(\mathrm{m} \cdot \mathrm{K})\right.$, $\left.\kappa_{\theta}^{\prime}=0.1 \mathrm{~W} /(\mathrm{m} \cdot \mathrm{K})\right)$. We can see that the temperature profiles bend toward the central region and the temperature gradient in the central region is enhanced greatly. More vivid comparison between the thermal cloak and the reverse thermal cloak can be found in Fig. 2. The reverse thermal cloak tends to concentrate the heat and have the most heat diffuse from it, resulting in local heating and large temperature gradient. Isothermal region forms in the thermal cloak while a sharp temperature drop arises in the reverse thermal cloak.

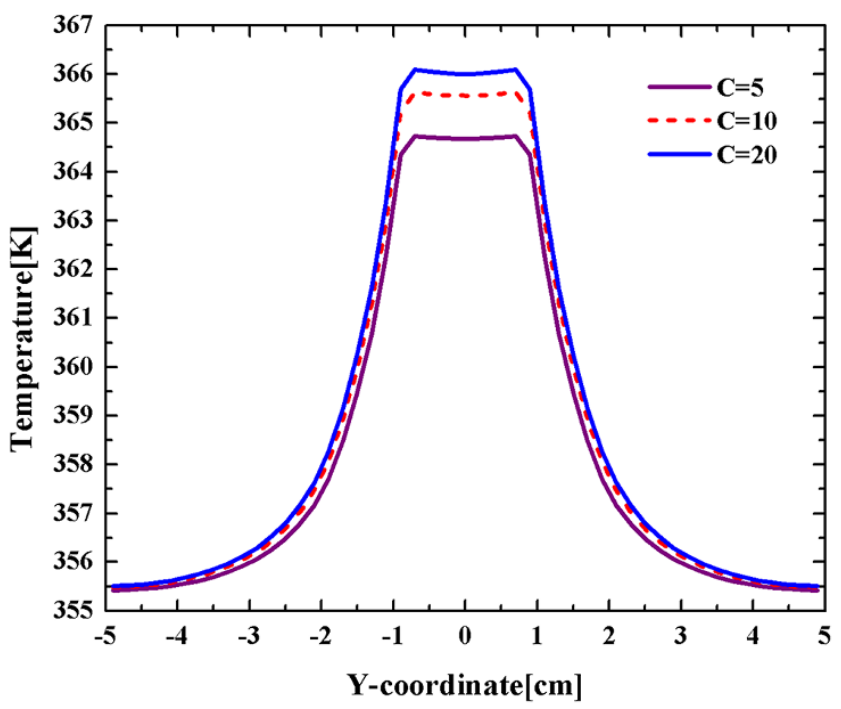

Figure $3 \mid$ Temperature profiles of a cut plane $(x=-0.5 \mathrm{~cm})$ on an annular reverse thermal cloak with changing the constant $C$ from 5 to 20 .
To further demonstrate the local heating phenomenon, we plotted the temperature curves on the cut plane $(x=-0.5 \mathrm{~cm})$ in Fig. 3. Obvious local high temperature can be observed in the central region. We can also see that when the constant $C$ increases from 5 to 20 , the local temperature in the central region is enhanced. The larger the constant $\mathrm{C}$ is, the larger the thermal conductivity anisotropy is. The trend in Fig. 3 implies that large anisotropy of the thermal conductivity distribution leads to enhanced local heating effect.

Transient simulations of the reverse thermal cloak were carried out and the heat diffusion processes are shown in Fig. 4 $\left(C=\kappa_{r}^{\prime}=10 \mathrm{~W} /(\mathrm{m} \cdot \mathrm{K}), \kappa_{\theta}^{\prime}=0.1 \mathrm{~W} /(\mathrm{m} \cdot \mathrm{K})\right)$. In the beginning, heat has not conducted to the right part yet and the isotherms squeeze in the left part. With time elapse, heat conducts to the right part gradually and the isotherms stretch to the whole domain. At $t=60$ second, the thermal system almost get its equilibrium state and the corresponding isotherms are symmetric in the left and right parts. No matter in the transient state (see Fig.4) or in the steady state (see Fig. 1 and Fig. 2), we can discover that the isotherms bend toward the central region in both the left and right parts. This implied that in the reverse thermal cloak, heat conducts faster in the left part and slower in the right part. As a result, compact isotherms and large temperature gradient form in the central region.

From above analyses, we can observe the local heating effect and there exists a large temperature gradient in the central region of the reverse thermal cloak. Since the driving force of heat diffuse is the temperature gradient, one idea is that we could utilize the large temperature gradient to heat up objects. To verify our idea, we built a three-dimensional model in which we modeled a substrate consisting of a reverse thermal cloak $\left(C=\kappa_{r}^{\prime}=10 \mathrm{~W} /(\mathrm{m} \cdot \mathrm{K}), \kappa_{\theta}^{\prime}=0.1 \mathrm{~W} /\right.$ $(\mathrm{m} \cdot \mathrm{K}))$ and then mounted a cubic probe on the central region, as shown in Fig. 5(a). The dimensions of the substrate are $10 \times 10 \times 0.5$ $\mathrm{cm}^{3}$. The dimensions of the cubic probe are $1 \times 1 \times 0.5 \mathrm{~cm}^{3}$. The interior and exterior radii of the annular reverse thermal cloak are $1 \mathrm{~cm}$ and $2 \mathrm{~cm}$, respectively. For comparison, we also built the samedimensional model except that the substrate is a homogeneous plane without reverse thermal cloak. After steady-state simulations, we selected the temperature contours on the front surface of the cubic probe (on the plane of $x=-0.5 \mathrm{~cm}$ ) to demonstrate our idea. The temperature comparisons are shown in Fig. 5(b) and Fig. 5(c). We can see that the maximum temperature of the cubic probe is $370 \mathrm{~K}$ when the substrate is with a reverse thermal cloak; the maximum temperature is $357 \mathrm{~K}$ when the substrate is without a reverse thermal cloak. Thus the reverse thermal cloak can be utilized to heat up objects.

To realize such a reverse thermal cloak whose radial thermal conductivity is smaller than its azimuthal thermal conductivity, we designed a composite structure made by alternating spoke-like layers of two homogeneous materials. As shown in Fig. 6, the structure consists of two isotropic materials, i.e. material A and material B. 


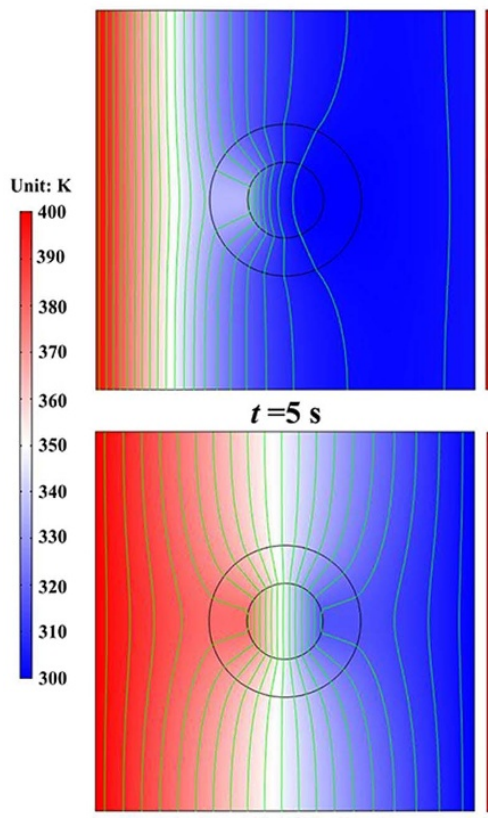

$t=30 \mathrm{~s}$

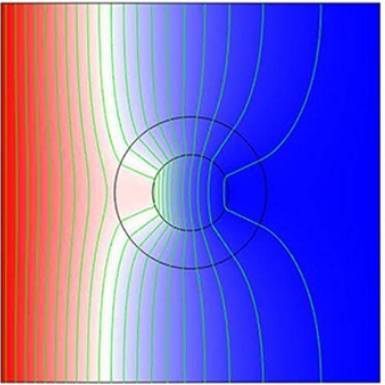

$t=10 \mathrm{~s}$

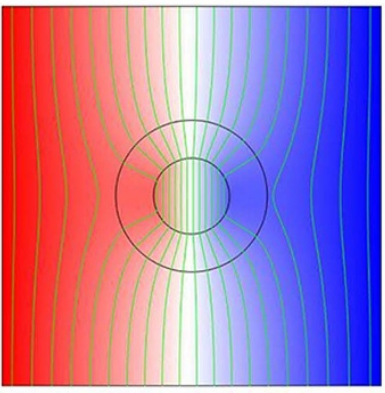

$t=40 \mathrm{~s}$

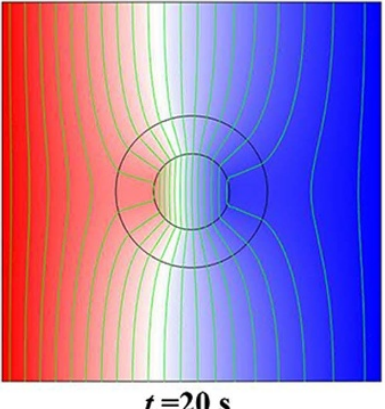

$t=20 \mathrm{~s}$

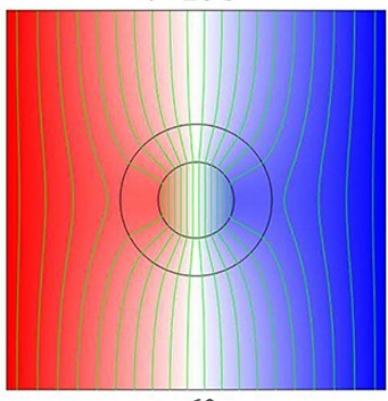

$t=60 \mathrm{~s}$

Figure $4 \mid$ Transient heat conduction through an annular reverse thermal cloak with the left and right boundaries at constant temperature. Snapshots of temperature profile with time elapse from $t=5 \mathrm{~s}$ to $\mathrm{t}=60 \mathrm{~s}$. Isotherms are illustrated with green color in panel.

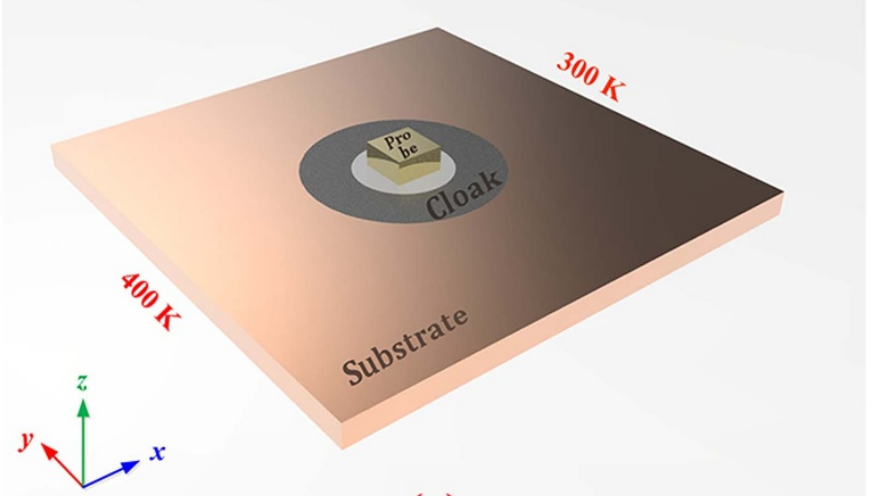

(a)

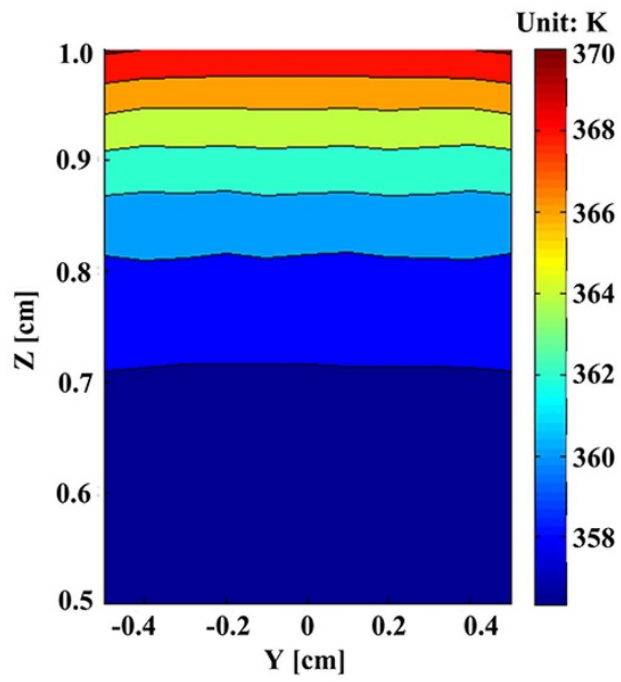

(b)

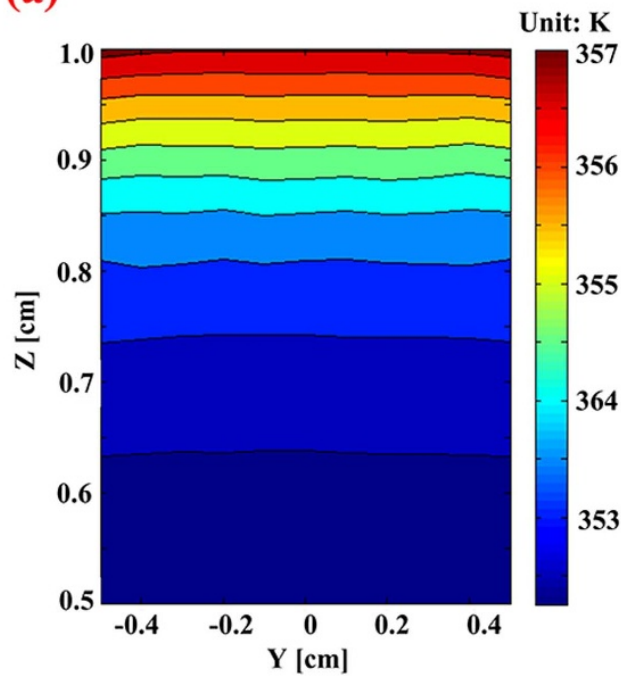

(c)

Figure $5 \mid$ (a) 3-D model of a substrate consisting of a reverse thermal cloak and a cubic probe, (b) temperature contours of the cubic probe on a reverse thermal cloak, and (c) temperature contours of the cubic probe on a homogenous plane with the same dimensions. 


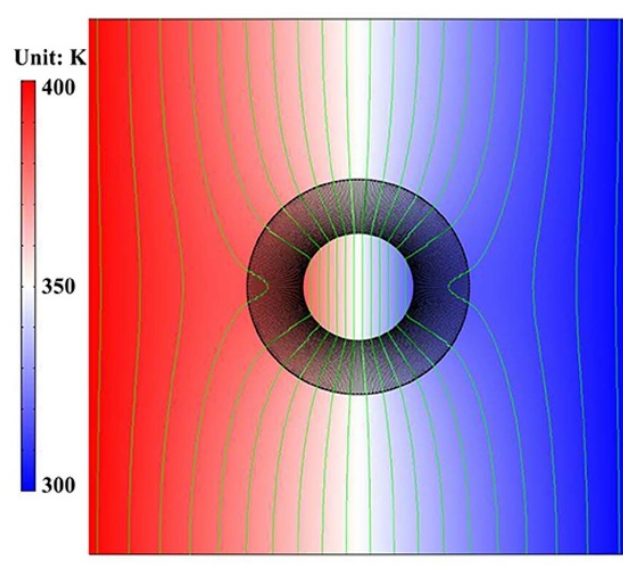

(a)

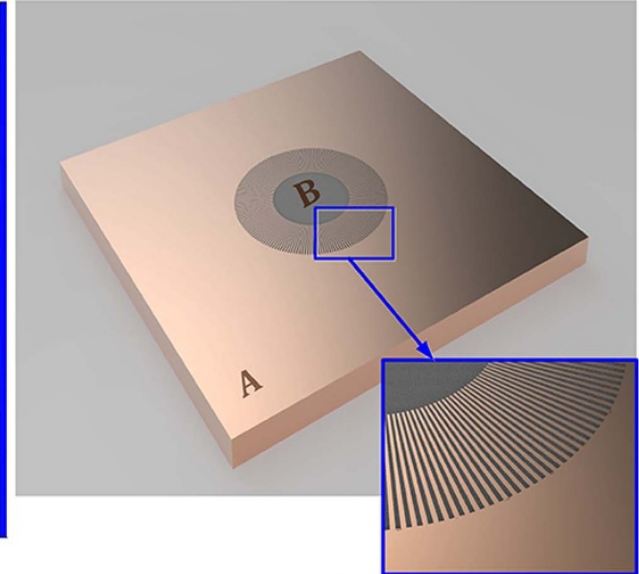

(b)

Figure 6 Simulation of a reverse thermal cloak consisting of alternating spoke-like layers of two homogeneous materials (A and B): (a) the temperature profile and (b) its structure.

The thermal conductivities of these two materials are $10 \mathrm{~W} /(\mathrm{m} \cdot \mathrm{K})$ and $0.1 \mathrm{~W} /(\mathrm{m} \cdot \mathrm{K})$, respectively. All the boundary conditions are kept the same. The simulated temperature profile is similar to above simulations where compact isotherms and local heating effect form in the central region. This implies that the reverse thermal cloak not only can be realized by pre-engineered metamaterials, but also can be realized by homogeneous materials with alternating spoke-like structures.

Another method to realize such kind of reverse thermal cloak with homogeneous materials can ascend to the Hashin coated sphere. According to the two-dimensional Hashin-Shtrikman formula ${ }^{14}$, the effective thermal conductivity $\kappa^{*}$ can be calculated as

$$
\kappa^{*}=\kappa_{2}+\frac{2 f_{1} \kappa_{2}\left(\kappa_{1}-\kappa_{2}\right)}{2 \kappa_{2}+f_{2}\left(\kappa_{1}-\kappa_{2}\right)}
$$

With

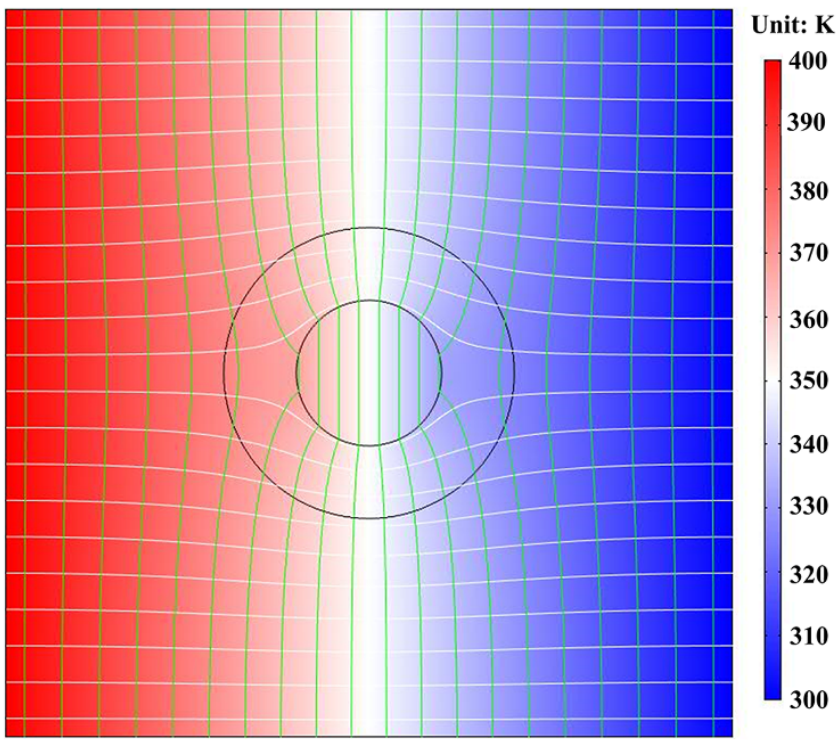

Figure $7 \mid$ Simulation of another reverse thermal cloak comprised of homogenous materials. The thermal conductivity of the reverse thermal cloak is $0.918 \mathrm{~W} /(\mathrm{m} \cdot \mathrm{K})$, which is designed according to the HashinShtrikman theory. The isotherms and streamlines are also illustrated with green and white colors in panel, respectively.

$$
f_{1}=1-f_{2}=\left(r_{1} / r_{2}\right)^{2}
$$

where $\kappa_{1}, \kappa_{2}$ are the thermal conductivities of the core region and the shell region, respectively. $f_{1}$ and $f_{2}$ are the two-dimensional volume fraction, $r_{1}$ and $r_{2}$ are the exterior radii of the circular core and the cloaking shell. When $\kappa^{*}$ equals to the thermal conductivity of the outside matrix $\kappa_{0}$, reverse thermal cloak can be realized.

According to dimensions and thermal conductivities mentioned above, the thermal conductivity of the cloaking shell $\kappa_{2}$ can be calculated as $0.918 \mathrm{~W} /(\mathrm{m} \cdot \mathrm{K})$ in this paper. With the same method, we simulated the temperature field and the result was plotted in Fig. 7. It is seen that the green isotherms and the white streamlines are orthometric to each other in panel. The isotherms bend to the core region and a large temperature gradient forms there. Therefore, with the Hashin-Shtrikman formula, we can design the dimensions and thermal conductivities to realize reverse thermal cloaks. But when comparing Fig. 7 with Fig. 4 and Fig. 6, it is also seen that the thermal gradient in Hashin coated sphere is not as compact as that realized by pre-engineered metamaterials, nor homogeneous materials with alternating spoke-like structures. Thus it is perceived that the local heating effect in Hashin coated sphere is not as strong as the other two, and the heat-concentration ability of Hashin coated sphere is also limited.

\section{Discussion}

In summary, we demonstrated in this study that reverse thermal cloak can be applied to realize local heating. Unlike the thermal cloak can realize isothermal region and render heat invisible from the outside temperature field, the reverse thermal cloak can bend the isotherms to the central region and realize large temperature gradient there. Due to the large temperature gradient, local heating phenomenon was observed. Such "local heating" phenomenon can be enhanced by re-distributing the dispersion of thermal conductivity in the domain of the reverse thermal cloak. Here, a way to realize local heating was proposed by the reverse thermal cloak. Like the fabrication of thermal cloak, the reverse thermal cloak can also be fabricated with pre-engineered metamaterial or even with homogenous materials by spoke-like structures or Hashin coated-sphere structure. With the reverse thermal cloak, we can realize the local heating in many fields. For instance, in the electronic packaging, with local heating, we may avoid heating some temperature-sensitive components or causing printed circuit board (PCB) warping.

It should be pointed out that the reverse thermal cloak was proposed to correspond to the thermal cloak during the research of 
thermal cloak. In this paper, we simply defined the difference between these two cloaks as that: for thermal cloak, the constant $C$ (= radial thermal conductivity $\kappa_{r}^{\prime}$ ) is less than 1 ; for the reverse thermal cloak, the constant $C$ is larger than 1 . The essence of both thermal cloak and reverse thermal cloak is the anisotropic dispersion of thermal conductivity. When compared with the thermal concentrator $^{15,18}$, the present reverse thermal cloak exhibits similar characteristics but in a much simpler engineering way. The thermal concentrator has specific formula to identify the dispersion of thermal conductivity ${ }^{15}$, while in this paper we extended the limitation of thermal conductivity and gave a general formula in Eq. (1). Moreover, we paid more attentions to the application of reverse thermal cloak, rather than just staying in the theoretical or laboratory stages. What should be emphasized is that the present reverse thermal cloak can also be applied to other shape cloaks, like square, rectangular, elliptic, etc.

\section{Methods}

When a temperature gradient exists in a body, experience has shown that there is an energy transfer from the high-temperature region to the low-temperature region. For a steady state without heat source, the control equation can be written as $\nabla(\kappa \nabla T)=0$, where $\kappa$ is the thermal conductivity and $T$ is the temperature. Similar to the invariance of Maxwell equations in the transformation optics, Guenneau et al. ${ }^{15}$ pointed out that the heat conduction equations can also be written in a form-invariant manner under coordinate transformations. Thus, in the transformed space, the heat conduction equation can be re-written as

$$
\nabla^{\prime}\left(\hat{\kappa} \nabla^{\prime} T^{\prime}\right)=0 .
$$

With

$$
\hat{\kappa}=\frac{\Lambda \kappa \Lambda^{\mathrm{T}}}{\operatorname{det}|\Lambda|}
$$

where $\Lambda$ is the Jacobian matrix with components defined as $\Lambda=\partial\left(x^{\prime}, y^{\prime}, z^{\prime}\right) / \partial(x, y, z)$. The Jacobian matrix characterizes the geometrical variation in the original space $(x, y, z)$ and the transformed space $\left(x^{\prime}, y^{\prime}, z^{\prime}\right)$. Eqs. (2) and (3) construct the basis of transformation thermodynamics and we can design and manipulate the flow of heat by an arbitrary coordinate transformation.

One seminal typical design of thermal cloak was consulted to the invisible cloak proposed by Pendry et al. ${ }^{1,10}$ With the simple coordinate transformation in Eq. (4), we can squeeze the heat flow from a central region $\left(r \leq R_{2}\right)$ in the original space $(r, \theta, z)$ into a shell region $\left(R_{1} \leq r \leq R_{2}\right)$ in the transformed space $\left(r^{\prime}, \theta^{\prime}, z^{\prime}\right)$, while the rest of heat flow (in the region $r>R_{2}$ ) are maintained.

$$
\left\{\begin{array}{l}
r^{\prime}=R_{1}+r\left(R_{2}-R_{1}\right) / R_{1} \\
\theta^{\prime}=\theta \\
z^{\prime}=z
\end{array}\right.
$$

where $R_{1}$ and $R_{2}$ are the interior and exterior radii of the annular thermal cloak. Substituting Eq. (6) into Eqs. (4) and (5) yields Eq. (1).

1. Pendry, J. B., Schurig, D. \& Smith, D. R. Controlling electromagnetic fields. Science 312, 1780-1782 (2006).

2. Leonhardt, U. Optical conformal mapping. Science 312, 1777-1780 (2006).
3. Zolla, F., Guenneau, S., Nicolet, A. \& Pendry, J. B. Electromagnetic analysis of cylindrical invisibility cloaks and the mirage effect. Opt. Lett. 32, 1069-1071 (2007).

4. Zhang, S., Xia, C. G. \& Fang, N. Broadband acoustic cloak for ultrasound waves. Phys. Rev. Lett. 106, 024301 (2011).

5. Zhu, J. et al. Acoustic rainbow trapping. Sci. Rep. 3, 1728 (2013).

6. Farhat, M., Enoch, S., Guenneau, S. \& Movchan, A. B. Broadband cylindrical acoustic cloak for linear surface waves in a fluid. Phys. Rev. Lett. 101, 134501 (2008).

7. Zhang, S., Genov, D. A., Sun, C. \& Zhang, X. Cloaking of matter waves. Phys. Rev. Lett. 100, 123002 (2008).

8. Brun, M., Guenneau, S. \& Movchan, A. B. Achieving control of in-plane elastic waves. Appl. Phys. Lett. 94, 061903 (2009).

9. Hashin, Z. The Elastic Moduli of Heterogeneous Materials. J. Appl. Mech. 29, 143-150 (1962).

10. Pendry, J. B., Aubry, A., Smith, D. R. \& Maier, S. A. Transformation optics and subwavelength control of light. Science 337, 549-552 (2012).

11. Liu, Y. M. \& Zhang, X. Recent advances in transformation optics. Nanoscale 4, 5277 (2012).

12. Kundtz, N. B., Smith, D. R. \& Pendry, J. B. Electromagnect design with transformation optics. Proc. IEEE 99, 1622-1633 (2011).

13. Hashin, Z. \& Shtrikman, S. A Variational Approach to the Theory of the Effective Magnetic Permeability of Multiphase Materials. J. Appl. Phys. 33(10), 3125-3131 (1962).

14. Milton, G. W. Assemblages of spheres, ellipsoids, and other neutral inclusions, The Theory of Composites 115-117 (Cambridge, New York, 2004).

15. Guenneau, S., Amra, C. \& Veynante, D. Transformation thermodynamics: cloaking and concentrating heat flux. Opt. Express 20, 8207-8218 (2012).

16. Han, T. C., Yuan, T., Li, B. W. \& Qiu, C. W. Homogenous thermal cloak with constant conductivity and tunable heat localization. Sci. Rep. 3, 1593 (2013).

17. Schittny, R., Kadic, M., Guenneau, S. \& Wegener, M. Experiments on transformation thermodynamics: molding the flow of heat. Phys. Rev. Lett. 110, 195901 (2013).

18. Narayana, S. \& Sato, Y. Heat flux manipulation with engineered thermal materials. Phys. Rev. Lett. 108, 214303 (2012).

19. Leonhardt, U. Cloaking of heat. Nature 498, 440-441 (2013).

\section{Acknowledgments}

The authors would like to acknowledge the financial support in part by Natural Science Foundation of China (51376070), and in part by 973 Project of the Ministry of Science and Technology of China (2011CB013105), and in part by National 863 Project of the Ministry of Science and Technology of China (2011AA03A109).

\section{Author contributions}

R.H., X.L.W., J.Y.H. and X.B.L. contributed in the discussion. R.H. and J.Y.H. participated in the simulation. R.H. prepared the manuscript. R.H. and X.B.L. conceived the idea and revised the manuscript. X.B.L. supervised the project.

\section{Additional information}

Competing financial interests: The authors declare no competing financial interests. How to cite this article: Hu, R., Wei, X.L., Hu, J.Y. \& Luo, X.B. Local heating realization by reverse thermal cloak. Sci. Rep. 4, 3600; DOI:10.1038/srep03600 (2014).

This work is licensed under a Creative Commons AttributionNonCommercial-NoDerivs 3.0 Unported license. To view a copy of this license, visit http://creativecommons.org/licenses/by-nc-nd/3.0 